\title{
LUNG CANCER DETECTION USING MARKER- CONTROLLED WATERSHED WITH SVM
}

\author{
Fatema Tuj Johora $^{1}$, Mehdi Hassan Jony ${ }^{2}$, Md. Shakhawat Hossain ${ }^{3}$ and Humayan Kabir \\ Rana $^{4}$
}

\begin{abstract}
Lung cancer is one of the most dangerous diseases and prediction of it, is the most challenging problem nowadays. Most of the cancer cells are overlapped with each other. It is hard to detect the cells but also essential to identify the presence of cancer cells in the early stage. Early detection of lung cancer may reduce the death rate. In this study, we used the Grey Level Co-occurrence Matrix (GLCM) to extract the feature of cancer affected lung image and then Support Vector Machine (SVM) has been used to detect normal and abnormal lung cells after implementing the features. Our experimental evaluation using MATLAB demonstrates the efficient performance of the proposed system and in the result.
\end{abstract}

Index Terms - Lung Cancer Detection, Gabor Filter, Computed Tomography, Marker-Controlled Watershed, GLCM, SVM.

\section{INTRODUCTION}

$\mathrm{L}$ lung cancer is the maximum mainstream cancer that cannot be neglected. It is the second most regularly analyzed cancer in men and women. An anticipated 234,030 new instances of lung cancer are normal in 2018, representing around $14 \%$ of all cancer analyze [1]. The rate has been declining since the mid-1980s in men, however just since the mid-2000s in women [1].

Gender orientation contrasts reflect authentic examples of smoking take-up and end in the course of recent decades. During 2005 to 2014, lung cancer

\footnotetext{
${ }^{1}$ This paper was received on 31 March 2019, revised 03 July 2019 and accepted on 15 July 2019. This work was supported financially by GUBJSE.

Fatema Tuj Johora is with the Department of Computer Science and Engineering, Green University of Bangladesh, Dhaka,

Bangladesh. E-mail: fatema@cse.green.edu.bd.

Mehdi Hassan Jony, is a Research Assistant with the The Hefei University of Technology, China. E-mail: mehdi@mail.hfut.edu.cn Md. Shakhawat Hossain is with the Department of Computer Science and Engineering, Green University of Bangladesh, Dhaka, Bangladesh. E-mail: shakhawat@cse.green.edu.bd.

Humayun Kabir Rana, is with the Department of Computer Science and Engineering, Green University of Bangladesh, Dhaka, Bangladesh. E-mail: humayan@cse.green.edu.bd.
}

Frequency rates decreased by $2.5 \%$ per year in men and $1.2 \%$ per year in women [1]. Lung cancer

represents a larger number of deaths than some other cancer in men and women [1].

An expected 154,050 deaths are relied upon to happen in 2018, representing around 1 of every 4 cancer death. The lung cancer death rate has decayed by $45 \%$ since 1990 in men and by $19 \%$ since 2002 in women due to reductions in smoking, with the pace of decline quickening over the past decade; from 2011 to 2015, the rate decremented by $3.8 \%$ per year in men and by $2.3 \%$ per year in women [1]. Identifying of lung cancer are often done through its symptoms embrace a cough, coughing of blood, fatigue, unexplained weight loss, shortness of breath, etc. Computer Tomography (CT) is the most effective technique for detecting lung nodules for the ability to create threedimensional (3D) photos of fungus, which is more widely researched about nodule and tumor pathology. CT images were widely used in the clinic by a computer process to enjoy pulmonary nodules medicine. In the computer-aided diagnosis (CAD) system, lung cancer symptoms (CAD) are often divided into identification methods (often abbreviated as CADe) and diagnostic systems (often abbreviated as CADx). The foreskin system divides candidate nodules into the previous step nodules or non-nodules (e.g. traditional physiological structure). The aim of the CADx system is to restore detected nodules to Benign and malignant nodules [2]. Because the possibility of malignant pulmonary is often associated with geometric shapes, shapes and looks, CADx will separate intelligent and deadly lung nodules by effective options such as texture, shape and measurement. Thus, the prosperity of the specific CADx system is measured in terms of measurement, speed and automatic level [3].

In this study, image enhancement, segmentation, feature extraction and lung nodules detection procedures have been implemented using MATLAB. The paper is aiming to get more accurate results by introducing a new technique feature extraction process which is much effective and less time- 
consuming to detect cancerous lung. In the first section, the methodology has been explained rests of the paper following contents are classification with SVM as well as result and discussions. And at the end, a summary of the works and future work scope has been explained in conclusion.

\section{RELATED WORKS}

S. Avinash et al [4] showed that early detection of lung cancer is applicable for efficient analysis and it will help the opticians to provide initial treatment. Prior to this, researchers employed methods such as fast Fourier transform (FFT) for image enhancement, thresholding method for segmentation and extrusion methods for feature extracting etc. The research has computerized these selections, enhancing database, selecting features of lung cancer images lung cancer detection, quality and accuracy improvement.

Image processing approaches brought a huge benefit for identifying lung cancer at an early stage. Magnetic resonance imaging (MRI), which produces computerized images of the body that are used to diagnose several different cancers, was developed by Paul Lautenberg and Peter Mansfield [5]. The imaging is based on the different signals obtained from different tissue types that are subjected to a high magnetic field. In 2003 the developers won the Nobel Prize in medicine for their work.

Radiologists have interior relied on examining images from chest radiography and PET scans to detect lung cancer [6]. Fatema et al. [7] proposed a technique based on CT technology, the proposed system consists of a few steps like photo acquisition, preprocessing, feature extraction and categorization. At the preprocessing stage, RGB images are converted to grayscale images, used for reducing the Gabor filter, the adaptive thresholding method transforms CT scan images into binary images, and the REGIONPROPS function is used for the right body, from the binary image to the region. Feature extraction platforms, such as contrast, correlation, energy, home genetics, are characterized by a static method called Gray Level Co-occurrence Matrix (GLCM). To ameliorate the hand annotation of nodules, medical experts expand beyond an axial scan mode and rely on other techniques such as maximum intensity projections and 3D volume renderings [8]. Maximum intensity projection (MIP) is a volume rendering technique for $3 \mathrm{D}$ images that projects voxels with a maximum intensity of the parallel rays from a given viewpoint onto the plane [9]. This technique makes it more facile to detect denser objects like nodules since maximum projections will be concentrated in a particular area, whereas other structures like thin blood vessels will have maximum intensities more distributed throughout the lung/image. In Prior al. [10] are based upon operating and maintaining a high-availability image archive is a complex challenge involving varied archive-specific resources and driven by the needs of both image submitters and image consumers. In this research describes the management tasks and user support model for TCIA.

In [11] the authors largely fixate on adequately great amelioration in contrast of masses along with the suppression of background tissues is acquired by processing the parameters of the proposed transformation function in the definite range. The manual analysis of the sputum samples is timeconsuming, erroneous and needs concentrated trained person to evade diagnostic mistakes. The segmentation results will be utilized as a base for a Computer Availed Diagnosis system for early detection of cancer, which increases the chances of patient survival. As well as Gabor filter is proposed by authors for the enhancement of medical images. It is a good enhancement to implement for medical images.

Thus, Researchers are becoming more and more concerned with the elaboration of automated CAD systems for lung cancer. Many publications proposed different automated nodule recognition systems utilizing image processing, and including, different techniques for segmentation, feature extraction, and relegation. Moreover, the detection processes work in the middle stage of lung cancer, which is very hazardous for the affected patient [12].

\section{METHODOLOGY}

The methodology focuses to obtain a more accurate

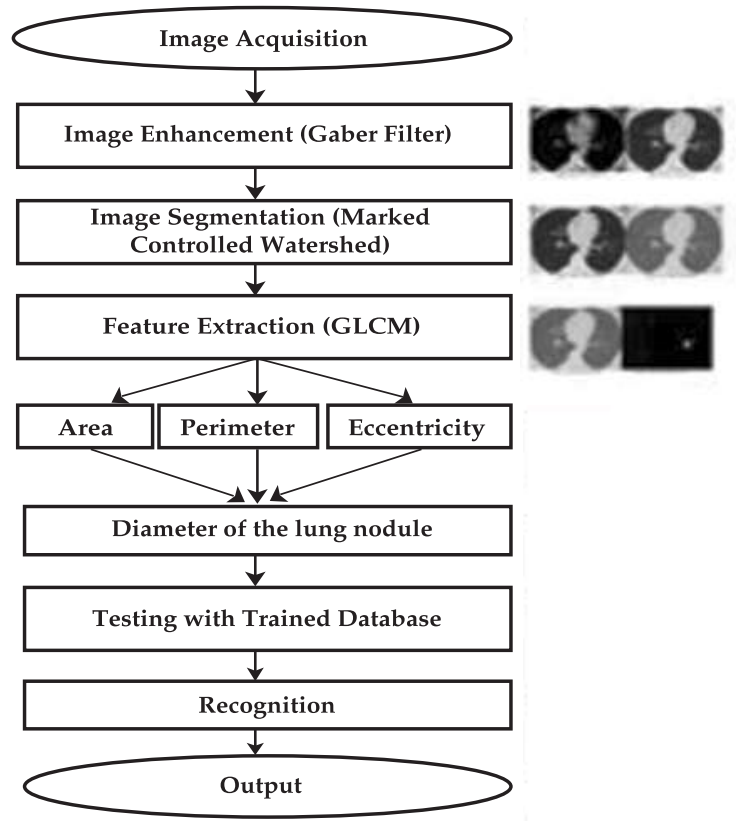

Fig. 1: Block diagram of the proposed system to detect lung cancer 
result. In the proposed method, it is enhancing the contrast of the input image through the pre-processing method. It is done by first converting the input image to a grayscale image and then it is modified with the Gabor filter to enhance [13].

Marker-Controlled Watershed technique has been used to segment the enhanced image. In feature extraction, a pixel comparing process is proposed. Lastly, we have used SVM for detecting lung nodules.

We use gabor filter for enhancement and markercontrolled watershed for segmentation because both are effective in both 2-D and 3-D, Fourier transform of the Gaussian function finds out more details [14], which can identify not only nodules but also some small details. Marker controlled is a colorful process which is effective in any modern image segmentation process to develop a robust methodology [15]. There are very few features extraction process like GLCM which can measure a specific area of an image. After measuring area, we discussed the size of the nodules with expert medical specialists. After watching the CT scan images and many of them told about the stages of the lung cancer.

\section{A. Image Acquisition}

TCIA uses a standards-based approach to nondetection of images stored in the Digital Imaging and Communication Medicine (DICOM) format to release protected health information (PHI). The reproduction and repetition of the measurement of the three radiologists were high (all $\mathrm{cc}, \geq 0.96$ ) [12]. The computer-aided measurement was more repetitive (all ccc, 1.00). There was a $95 \%$ limit for computer-aided unidimensional, two-dimensional, and twodimensional scanning volume measurement $(-7.3 \%$, $6.2 \%),(-17.6 \%, 19.8 \%)$, and $(-12.1 \%, 13.4 \%)$ respectively [12]. Chest CT scans are well reproducible. Unusual wound size exceeds the variation of measurement of the computer system in size of $8 \%$ [12] or more and can be considered as significant when estimating the results of patient therapy. The whole-lung dataset consists of 15,419 CT scans obtained in a single breath hold with a 1.25 $\mathrm{mm}$ slice thickness. It is a public database [12]. DICOM (Digital Imaging and Communication in Medicine) has become a standard for medical imaging.

The acquired image is in raw form. A lot of noise in the acquired image is observed. To improve contrast, transparency, background sound separation, it needs to be pre-processed. Therefore, different techniques like enhancement, the required form is developed from images.

\section{B. Image Enhancement}

The image pre-processing stage commences with image enhancement. The aim of image enhancement is to modernize the interpretability or cognizance of information included in the image for human viewers or to provide better input for other automated image processing techniques. Image enhancement techniques can be divided into two broad categories: Spatial domain methods and frequency domain methods [16].

- Spatial domain techniques- which operate directly on pixels.

- Frequency domain techniques- which operate on the Fourier transform of an image.

The various image enhancement techniques can be characterized as spatial domain methods and frequency domain methods. This includes smoothing of image and removal of noises, blurring etc. There are many ways to enhance the image but Gabor filter was found to be suitable for both the CT and MRI images. In the image enhancement stage, we utilized the following Gabor filter techniques.

\section{Gabor Filter}

A Gabor filter is a linear filter whose impulse replication is defined by a harmonic function multiplied by a Gaussian function. Because of the multiplication-convolution property, the Fourier transform of a Gabor filter's impulse replication is the convolution of the Fourier transform of the harmonic function and the Fourier transform of the Gaussian function [14]. It fundamentally analyses whether there is any concrete frequency content in the image in concrete directions in a localized region around the point or region of analysis.

A set of Gabor filters with different frequencies and orientations may be helpful for extracting useful features from an image. In the discrete domain, 2D Gabor filters are given by,

$$
\begin{aligned}
& \mathrm{G}_{\mathrm{c}}[i, j]=\mathrm{B} e^{\frac{i^{2}+j^{2}}{2 \alpha^{2}}} \cos 2 \pi f(i \cos \theta+j \sin \theta) \\
& \mathrm{G}_{\mathrm{s}}[i, j]=\mathrm{C} e^{\frac{i^{2}+j^{2}}{2 \alpha^{2}}} \sin 2 \pi f(i \cos \theta+j \sin \theta)
\end{aligned}
$$

Where $\mathrm{B}$ and $\mathrm{C}$ are normalizing factors to be determined. 2-D Gabor filters have rich applications in image processing, especially in feature extraction for texture analysis and segmentation. $f$ defines the frequency being looked for in the texture. By varying $\theta$, we can look for texture oriented in a particular direction. By varying $\alpha$, we change the support of the basis or the size of the image region being analyzed shown in fig 2 .

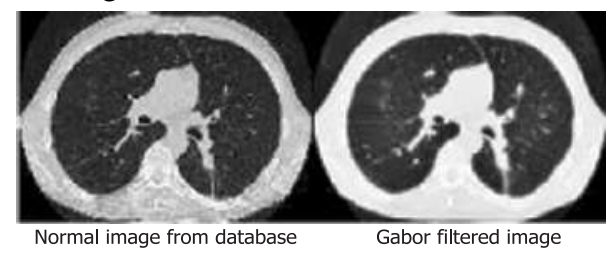

Fig. 2. Enhanced image by using Gabor filte 
TABLE I

ALGORITHM MARKER-CONTROLLED WATERSHED

\begin{tabular}{|c|c|}
\hline Procedure & Marker-Controlled Watershed \\
\hline Step 1 & $\begin{array}{l}\text { Calculate a segmentation function. This is an } \\
\text { image in which we are trying to segment them } \\
\text { with dark areas. }\end{array}$ \\
\hline Step 2 & $\begin{array}{l}\text { Counting foreground markers. This is attached } \\
\text { to the blobs of pixels in each object. }\end{array}$ \\
\hline Step 3 & $\begin{array}{l}\text { Counting background markers. This pixel is not } \\
\text { part of an object. }\end{array}$ \\
\hline Step 4 & $\begin{array}{l}\text { Modify the segmentation function so that it only } \\
\text { has minima at the front and background marker } \\
\text { position. }\end{array}$ \\
\hline Step 5 & $\begin{array}{l}\text { Calculate the watershed transform of the revised } \\
\text { segmentation function. }\end{array}$ \\
\hline
\end{tabular}

\section{Image Segmentation}

Image segmentation is to facilitate the representation of an image into something that is more meaningful and easier to analyze. Image segmentation is used to allocate objects and boundaries. It is the process of assigning a label to every pixel in an image such that pixels with the same label share certain visual characteristics [17]. The result of image segmentation is a set of segments that collectively cover up the entire image or a set of contours extracted from the image. Each pixel of the image in a region is similar with respect to some characteristic or computed property, such as color, intensity, or texture: adjacent regions are significantly different with respect to the same characteristics [18].

In this research, we used the marker-controlled watershed image segmentation approach:

\section{Marker-Controlled Watershed Approach}

The Watershed transformation considers the gradient enormity of an image as a topographic surface. Pixels having the highest gradient enormity intensities (GMIs) match up to watershed lines, which represent the region boundaries. Water placed on any pixel roofed by a flow downwards to a common local intensity minimum (LMI). Pixels draining to a common minimum form a catchment basin, which signify the regions. Image segmentation result is a set of segments that cover a set of contours that are fully outlined in the whole image [15]. The markercontrolled pond section follows these basic methods and shows such fig 3 .

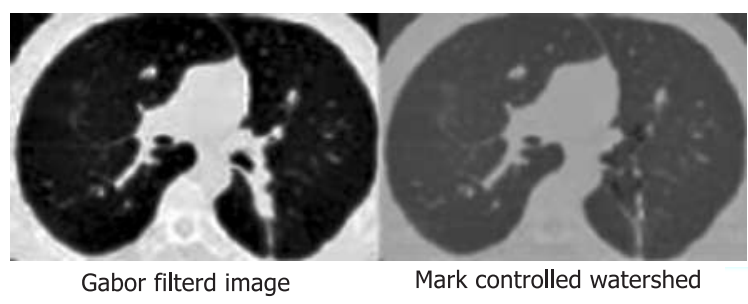

Fig. 3. Enhanced image to segmented

In segmentation, images are been segmented in various sections which made easy for extraction features with GLCM process. With watershed segmentation, we detected the unwanted nodules with abnormal pixels. The outlined of abnormal pixels positions with light violet color are very similar with given position (affected cell with nodules) which is obtained from the database.

\section{Feature Extraction}

In image processing, the Image features extraction stage is very vital image processing techniques which utilizing algorithms and techniques to detect and isolate portions or shapes (features) of an image $[19,20]$. Once the segmentation is accomplished in the lung region, the features can be obtained from it and the diagnosis rule can be designed to precisely detect the cancer nodules in the lungs. This diagnosis rules can eliminate detection of cancer nodules resulted in

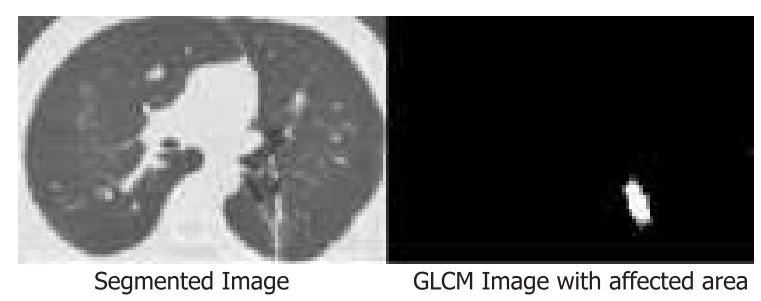

Fig. 4. Feature extraction from segmentation

segmentation and provides a better diagnosis [21-23].

In this stage to obtain the general features of the enhanced segmented image, GLCM is used as shown in fig 4 .

\section{Grey Level Co-Occurrence (GLCM)}

For extracting feature using the Gray-level cooccurrence Matrix (GLCM) to represent a set of features to reduces the misclassification of the glaucoma image. GLCM describes how many coordinates of pixel brightness values in an image illustration. GLCM represents the combined frequency of all pairs of gray levels $i$ and $j$ divided by a distance in the direction of nodules. GLCM Characteristics of basic character areas, perimeter, and Eccentricity this scale is measured in. These features are defined as:

A) Area: This is a scalar value that gives the actual number of actual pixels in extracted ROI. The Transformation function creates an array of ROI, which contains pixels with 255 values.

Area: $\mathrm{R}=\left(\mathrm{R} x, y, \mathrm{I}_{\mathrm{ROI}}[\right.$ Area $]=\mathrm{x}, \mathrm{J}_{\mathrm{ROI}}[$ Area $\left.]=\mathrm{y}\right)$

Where, $i, j$ are pixels in size within the shape. ROI is Interest Area. I ROI [] is vector contain ROI I position, J ROI [] vector retains ROI J position [24].

B) Perimeter: This is a scalar value that actually gives nodule pixels. It extracted ROI borders 
length. Transform function creates an array on the edge of which the pixels contain 255 values, at least one pixel that contains 0 values [22].

Perimeter: $\mathrm{C}=\left(\mathrm{C}_{\mathrm{x}, \mathrm{y}} \mathrm{I}_{\text {edge }}[\mathrm{C}]=\mathrm{x}, \mathrm{J}_{\text {edge }}[\mathrm{C}]=\mathrm{y}\right)$ Where $I$ is the edge [] and $J$ is the edge [] The vector represents the combination of $\mathrm{x}^{\text {th }}$ and $\mathrm{y}^{\text {th }}$ pixels by creating curves respectively.

C) Eccentricity: This metric value is called as roundness or circularity or irregular complex (I) equal to 1 for only round and it is less than 1 for any other shape.

Eccentricity $=\frac{\text { Length of Major Axis }}{\text { Length of Minor Axis }}$

Approximate features for a separate nodule of given sample images mentioned in Fig. 5 were found as follows:

1) Area: It is the simplest property and its given

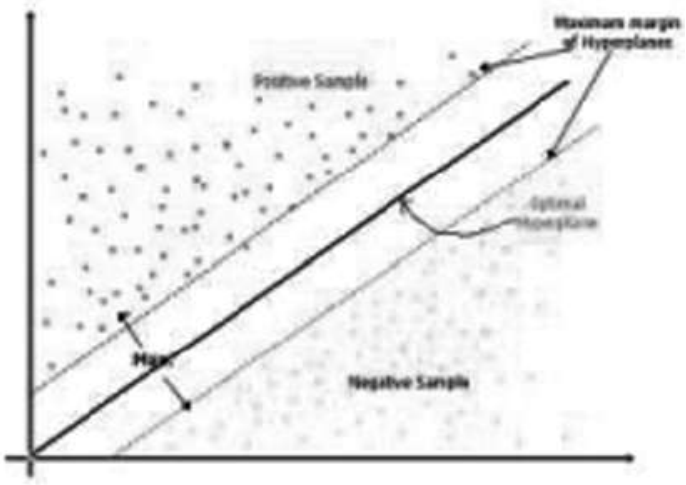

Fig. 5. SVM classifier hyperplanes

size. Therefore, it is the total number of white pixels in the extracted area.

2) Perimeter: Another simple property defined by the scope of this region. It's external ROI borders length.

3) Eccentricity: It is used to decide the shape or circularity of the object.

Area: 2291 Perimeter: 221 Eccentricity: 0.8289 Stage 3 Detected

\section{CLASSIFICATION}

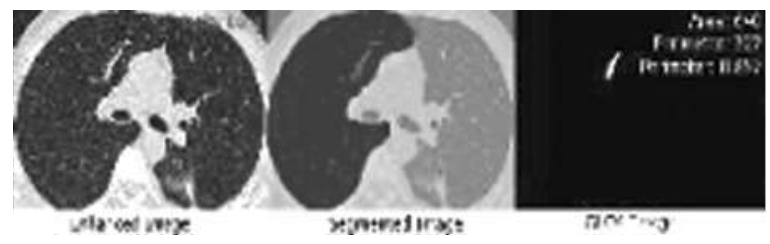

Fig. 6. A healthy lung test

Support vectors are a learning model that is supervised by the relevant learning algorithms that can identify data analyzes and patterns for machine analysis. The basic SVM input takes a set of data and for each given input, predicts that the two classes

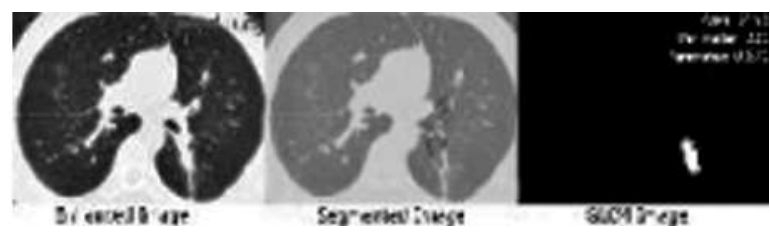

Fig. 7. A "stage 3" affected lung

form the input, it creates a non-possible binary linear classifier. SVM uses a kernel function that maps the given information to an individual location. Separation can be made with very complex boundaries. The various types of kernel functions, including polynomial, RBF, quadratic, Multi-Layer Perceptron (MLP). Each kernel is formulated by its own parameter $\gamma, \sigma$, and so on. Fig 5 shows the maximum margin hyperplane. The main hyper-plane algorithm is a way of creating non-linear classifications using the most margin hyper-plan kernel trick.

\section{RESULT AND DISCUSSION}

\section{A. Stage of Lung Cancer}

The lung nodules are the smallest increase in the lungs, which measure from $5 \mathrm{~mm}$ to $25 \mathrm{~mm}$. The unusual nodule size in images is more than $25 \mathrm{~mm}$.

Assessing the size of cancer in staging and entering the tissue surrounding it, as well as the presence or absence of metastasis in lymph nodes or other organs [25]. The stage from I to IV according to the intensity:

1. Stage I: cancer is confined to the lung

2. Stage II and III: cancer is confined to the chest

3. Stage IV: Cancer has spread from the chest to other parts of the body.

This experimental information consists of 15,419 lung images. These 15,419 lung images are passed through the proposed method. Then the rules of diagnosis are generated from those images and these rules are sent to SVM classifier for the learning process. After learning, a lungs image is passed on to the proposed method. Then the proposed method has processed through its processing steps and finally, it can detect whether the following lung image is cancerous or not which are shown in fig $6 \& 7$.

\section{B. Gaussian Radial Basis Function}

Radial basis functions kernel has received significant attention, most commonly with a Gaussian of the form,

$$
\mathrm{K}\left(\mathrm{a}, \mathrm{a}^{\prime}\right)=\exp \left(-\frac{\left|\mathrm{a}+\mathrm{a}^{\prime}\right|^{2}}{2 \mathrm{a}^{2}}\right)
$$

Generally, a method of clustering is first employed to select a subset of the center. An interesting feature of SVM is that this selection is perfect, each support vector centered on that data point, contributing to the 
local Gaussian function. The basic equation for support vector machines is:

$$
C_{0}=H^{T} \times F_{0}+b \ldots(7)
$$

Where, $\mathrm{C}_{0}=$ Output Class; $\mathrm{H}=$ Hyper-plane; $\mathrm{F}_{\mathrm{o}}=$ Input Feature; $b=$ Bias.

Now the dataset success rate is being compared with Prior al. [10] and Makaju al. [13] which has the same dataset with threshold binarization process. Patient one is the result with the same patients' MRI analysis report is done by this paper and subject two is the result with the same patient done by the following papers has shown in table 1.

Table 1: Comparing table with related works

\begin{tabular}{|l|l|l|l|l|}
\hline Patient & Type & Prior & Makaju & $\begin{array}{l}\text { Our } \\
\text { proposed }\end{array}$ \\
\hline \multirow{2}{*}{2073083717} & TAR & 88.23 & 83.23 & 93.23 \\
\cline { 2 - 5 } & FAR & 11.77 & 16.77 & 6.77 \\
\hline 1825099523 & TAR & 91.63 & 86.12 & 93.02 \\
\cline { 2 - 5 } & FAR & 8.37 & 13.88 & 6.98 \\
\hline 1867199702 & TAR & 90.96 & 84.32 & 88.47 \\
\cline { 2 - 5 } & FAR & 9.04 & 15.68 & 11.53 \\
\hline 1225316081 & TAR & 88.67 & 84.52 & 92.31 \\
\cline { 2 - 5 } & FAR & 11.33 & 15.48 & 7.69 \\
\hline \multirow{2}{*}{1286684383} & TAR & 89.59 & 85.63 & 89.91 \\
\cline { 2 - 5 } & FAR & 10.41 & 14.37 & 10.09 \\
\hline Total Success & Rate & 89.816 & 84.764 & 91.388 \\
\hline
\end{tabular}

\section{CONCLUSIONS}

Lung cancer is one of the most dangerous diseases in the world. Proper diagnosis and early detection of lung cancer can increase the survival rate. Current strategies include X-ray, CT scan, MRI, PET image study. Expert medical specialists diagnose and diagnose cancer levels by experience. Treatment includes surgery, chemotherapy, radiation therapy, and target therapy. This treatment is long, costly and painful. So, trying to a molecule in this method to detect lung cancer by using image processing techniques. CT scan images are acquired from different hospitals. These images include fewer words than X-ray and MRI images. An image improvement technique is developing for the detection and treatment stage of the disease before; The time factor is taking into account the discovery of abnormalities in the target image. CT scan captured images are processed. The field of interest, such as a tumor, is accurately identified from the original. Gabor Filters and Marker-Controlled Watershed segmentation gives the best results in the pre-processing phase. From the arising regions of interest, three properties are extracted, such as area, measurement, and warmth. These three properties help to detect lung cancer levels. The result indicates the different levels of the tumor. By measuring tumor levels, lung cancer stage can be correctly identified using the proposed procedure. The results show good prospects for lung cancer detection at an early stage. After using our methodology, cancer stage can be measured according to the size of the nodules. Doctors can define the stages.

Also, for the purpose of classification, support vector machine modeling is an attractive approach. They combine generalization controls with a strategy to address dimension curses. Kernel mapping enables a comparable comparison of commonly designated model architectures, providing a compact structure. In the classification problems, the generalization control is achieved by maximizing the margin, which is consistent with the weights of the weight vector in a canonical structure.

\section{REFERENCES}

[1] A. Staff, "Cancer Facts \& Figures 2018," Atlanta: American Cancer Society, Cancer, pp. 19-20, 2018.

[2] L. Sali, S. Delsanto, D. Sacchetto, L. Correale, M. Falchini, A. Ferraris, G. Gandini, G. Grazzini, F. Iafrate, G. Iussich, L. Morra, A. Laghi, M. Mascalchi and D. Regge, "Computerbased self-training for CT colonography with and without CAD”, European Radiology, 2018.

[3] Q. Song, L. Zhao, X. Luo and X. Dou, "Using Deep Learning for Classification of Lung Nodules on Computed Tomography Images", Journal of Healthcare Engineering, vol. 2017, pp. 17, 2017.W.-K.Chen, Linear Networks and Systems. Belmont, CA: Wadsworth, 1993, pp. 123-135.

[4] S. Avinash, K. Manjunath and S. Kumar, "An improved image processing analysis for the detection of lung cancer using Gabor filters and watershed segmentation technique", 2016 International Conference on Inventive Computation Technologies (ICICT), 2016. Available: 10.1109/inventive.2016.7830084 [Accessed 24 January 2019].

[5] T. Jones, D. Duquette, M. Underhill, C. Ming, K. Mendelsohn-Victor, B. Anderson, K. Milliron, G. Copeland, N. Janz, L. Northouse, S. Duffy, S. Merajver and M. Katapodi, "Surveillance for cancer recurrence in long-term young breast cancer survivors randomly selected from a statewide cancer registry", Breast Cancer Research and Treatment, vol. 169, no. 1, pp. 141-152, 2018.

[6] P. Reid and S. Walter, "Yield from a fast track referal system for radiologists suspecting lung cancer", Lung Cancer, vol. 115, p. S16, 2018.

[7] F. Tuj Johora, M. Jony and P. Khatun, "A New Strategy to Detect Lung Cancer on CT Images", International Research Journal of Engineering and Technology (IRJET), vol. 05, no. 12, pp. 27-32, 2018.

[8] I. Valente, P. Cortez, E. Neto, J. Soares, V. de Albuquerque and J. Tavares, "Automatic 3D pulmonary nodule detection in CT images: A survey", Computer Methods and Programs in Biomedicine, vol. 124, pp. 91-107, 2016.

[9] S. Masrochah, "Relationship between Slice Thickness to Artery Coronary Diagnostic Information on the Reconstruction of Maximum Intensity Protection (MIP)", Journal of Medical Science and clinical Research, vol. 05, no. 06, pp. 23140-23145, 2017.

[10] F. Prior et al., "The public cancer radiology imaging collections of The Cancer Imaging Archive", Scientific Data, vol. 4, p. 170124, 2017. Available: 10.1038/sdata.2017.124.

[11] H. Chien and D. Mackay, "How much complexity is needed to simulate watershed streamflow and water quality? A test combining time series and hydrological models", Hydrological Processes, vol. 28, no. 22, pp. 5624-5636, 2013.

[12] R. Lung CT, "The Cancer Imaging Archive (TCIA) Public Access - Cancer Imaging Archive Wiki", Wiki.cancerimagingarchive.net, 2019. [Online]. Available: 
https://wiki.cancerimagingarchive.net/display/Public/RIDER+ Lung+CT\#4097ffced7f64f32a18885ceffdf7b86. [Accessed: 23- Mar- 2019].

[13] S. Makaju, P. Prasad, A. Alsadoon, A. Singh and A. Elchouemi, "Lung Cancer Detection using CT Scan Images", Procedia Computer Science, vol. 125, pp. 107-114, 2018. Available: 10.1016/j.procs.2017.12.016.

[14] S. Avinash, K. Manjunath and S. Kumar, "An improved image processing analysis for the detection of lung cancer using Gabor filters and watershed segmentation technique", 2016 International Conference on Inventive Computation Technologies (ICICT), 2016.

[15] M. Firmino, G. Angelo, H. Morais, M. Dantas and R. Valentim, "Computer-aided detection (CADe) and diagnosis (CADx) system for lung cancer with likelihood of malignancy", BioMedical Engineering OnLine, vol. 15, no. 1, 2016.

[16] H. Rutika r, "Automated detection and diagnosis from lungs CT scan images", International Journal of Emerging Technologies and Innovative Research (www.jetir.org UGC and issn Approved), ISSN:2349-5162, vol. 2, no. 3, pp. pp785-787, 2015.

[17] H. Ren, Y. Zhou and M. Zhu, "Tree Image Segmentation Based on an Improved Two-Dimensional Otsu Algorithm", International Journal of Hybrid Information Technology, vol. 9, no. 9, pp. 199-210, 2016.

[18] Y. Zhang, H. Guo, F. Chen and H. Yang, "Weighted kernel mapping model with spring simulation based watershed transformation for level set image segmentation", Neurocomputing, vol. 249, pp. 1-18, 2017.

[19] Rana HK., Azam MS. and Akhtar MR. "Iris Recognition System Using PCA Based on DWT", SM Journal of Biometrics \& Bio-statistics, vol. 2, no. 3, 2017.

[20] Rana HK, Azam MS, Akhtar MR, Quinn JMW, Moni MA., "A fast iris recognition system through optimum feature extraction", PeerJ Preprints 7:e27363v2 https://doi.org/10.7287/peerj.preprints.27363v2, 2019.

[21] Y. Yuan, J. Wang, B. Li and M. Meng, "Saliency Based Ulcer Detection for Wireless Capsule Endoscopy Diagnosis", IEEE Transactions on Medical Imaging, vol. 34, no. 10, pp. 20462057, 2015.

[22] X. Jia and M. Meng, "A deep convolutional neural network for bleeding detection in Wireless Capsule Endoscopy images", $201638^{\text {th }}$ Annual International Conference of the IEEE Engineering in Medicine and Biology Society (EMBC), 2016.

[23] S. Suman, F. hussin, A. Malik, K. pogorelov, M. Riegler, S. Ho, I. Hilmi and K. Goh, "Detection and Classification of Bleeding Region in WCE Images using Color Feature", Proceedings of the 15th International Workshop on ContentBased Multimedia Indexing - CBMI '17, 2017.

[24] Suzuki K., "False-positive Reduction in Computer-aided Diagnostic Scheme for Detecting Nodules in Chest Radiographs", Academic Radiology, Volume 13, Number 10, pp.10-15, February 2005.

[25] G. Aviram and M. Revel, "Misclassification of Lymph Nodes in Lung Cancer Staging", Chest, vol. 151, no. 4, pp. 733-734, 2017.

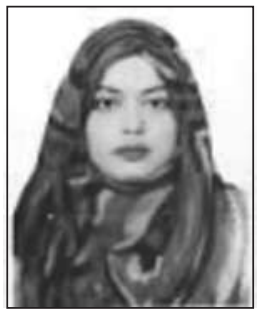

Fatema Tuj Johora was born in Dhaka, in 1995. She received her B.Sc degree in Computer Science and Engineering from Green University of Bangladesh, in 2017. She is currently a contractual Lecturer of Computer Science \& Engineering Department in Green University of Bangladesh. Her research interests include privacy and security in cloud database, medical image processing, bioinformatics etc. Now Fatema is working on watermarking in streaming database.

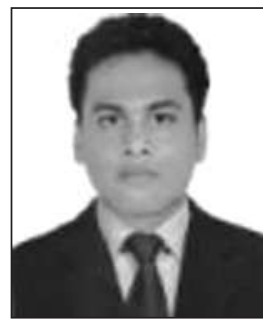

Mehdi Hassan Jony was born in Chittagong, Bangladesh, in 1994. He received the B.Sc degree in Computer Science engineering from the Green University of Bangladesh, in 2017 and studying M.S. degree in Computer Science engineering from the Hefei University of Technology. From 2017, he is a Research Assistant with the The Hefei University of Technology. His research interests include IoT, Artificial Intelligence, Cyber Security. Now-a-days, he is researching on watermarking in streaming database.

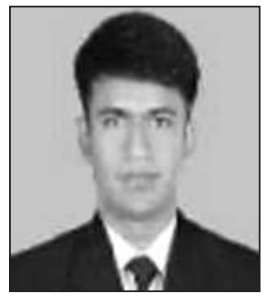

Md. Shakhawat Hossain received his B.Sc degree in Computer Science and Engineering from Green University of Bangladesh, in 2018. From 2018, he is a Research Assistant of Prof. Dr. Md. Abdur Razzaque, Associate Editor, IEEE Access, Editor, Elsevier's Journal of Network and Computer Applications. He is currently a contractual Lecturer of Computer Science \& Engineering Department in Green University of Bangladesh. His research interests include Networking, Cloud and Mobile Cloud Computing, IoT, Artificial Intelligence.

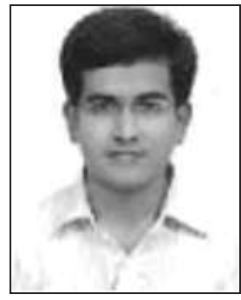

Humayan Kabir Rana received his B.Sc. Engineering degree in Computer Science and Engineering (CSE) from Pabna University of Science and Technology, in 2015. $\mathrm{He}$ is currently working as a Sr. Lecturer of CSE Department in Green University of Bangladesh. His research interests include Bioinformatics, Machine Learning, Digital Image Processing and Signal Processing. 